\title{
In vitro immunization approach to generate specific murine monoclonal IgG antibodies
}

Sophia Michelchen ${ }^{1}$, Burkhard Micheel ${ }^{1}$, Katja Hanack ${ }^{1,2}$

${ }^{1}$ Chair of Immunotechnology, Institute of Biochemistry and Biology, University of Potsdam, Karl-Liebknecht-Str. 24-25, D-14476 Potsdam, Germany

2 corresponding author. Tel.: +493319775348

E-mail addresses:

sophia.michelchen@uni-potsdam.de

burkhard.micheel@uni-potsdam.de

katja.hanack@uni-potsdam.de

\section{Abbreviations}

CFSE, carboxyfluorescein succinimidyl ester; DC, dendritic cell; ELISA, enzyme-linked immunosorbent assay; FCS, fetal calf serum; HRP, horse raddish peroxidase; Ig, immunoglobulin; IL, interleukin; LPS, lipopolysaccharide; NCS, neonatal calf serum; RT, room temperature; VP1, hamster polyomavirus capsid protein 


\section{Abstract}

Generating monoclonal antibodies to date is a time intense process requiring immunization of laboratory animals. The transfer of the humoral immune response into in vitro settings shortens this process and circumvents the necessity of animal immunization. However, orchestrating the complex interplay of immune cells in vitro is very challenging. We aimed for a simplified approach focusing on the protagonist of antibody production: the $B$ lymphocyte. We activated purified murine B lymphocytes in vitro with combinations of antigen and stimuli. Within ten days of culture we induced specific IgM and IgG antibody responses against a viral coat protein. Permanently antibody-producing hybridomas were generated. Furthermore we used this method to induce a specific antibody response against Legionella pneumophila. We thus established an effective protocol to generate monoclonal antibodies in vitro. By overcoming the necessity of in vivo immunization it may be the first step towards a universal strategy to generate antibodies from various species.

\section{Keywords}

monoclonal antibody, hybridoma technology, in vitro immunization, B cell activation 


\section{Introduction}

Antibodies are important tools in biotechnology and medicine with various applications in research, diagnosis and also increasingly in therapeutics (Geskin, 2015). In the organism a complex interplay of dendritic cells (DCs), T lymphocytes and B lymphocytes results in a polyclonal antibody response upon pathogen encounter. Whereas in vivo the resulting heterogenous mixture of antibodies in the serum is crucial for the clearance of an immunogen, the biotechnological usage often requires monoclonal antibodies with preferably high affinity and specificity for an antigen of choice. Generating such an antibody to date is a time and labor intensive process.

The hybridoma technique as one of the most widely used methods for antibody production utilizes the in vivo immune reaction of a laboratory animal upon immunization. The animal is challenged several times with the antigen of choice. This immunization comprises several weeks and also requires a considerable amount of antigen. Once a satisfying serum titer is reached the animal's spleen cells are fused with myeloma cells to generate stable hybridoma cell lines that secrete antibodies specific for the administered antigen. Immunization of the living organism and being dependent on spleen cells obviously limits this method to certain species with regards to ethical issues and feasibility. Furthermore, immunizing with highly immunogenic antigens may even cause premature death in laboratory animals.

Therefore, the immune reaction taking place in vivo does not leave any opportunity to intervene and underlies a multiplicity of biological factors. To circumvent and reduce these disadvantages a transfer to in vitro settings would be favourable. The transfer of antigen-specific immune responses to in vitro conditions has first been proposed by Mishell and Dutton in 1966 who induced an antibody response of unimmunized spleen cells. In 1988 Borrebaeck et al. published a general method to produce monoclonal antibodies from human lymphocytes in vitro. There have since been several approaches and protocols to induce antigen specific immune responses in vitro with varying success and reproducibility. Successful attempts as shown by Wand et al. (2011), Kato et al. (2012) and Inagaki et al. (2013) implied using splenocytes or a combination of DC, $T$ and B lymphocytes leading to the production of specific antibodies in vitro. This method allows monitoring of the process while minimizing the time and amount of antigen necessary for specific antibody production. It therefore offers several advantages compared to the common in vivo immunization. However, this procedure still involves a number of different cell types that have to be specifically activated and thus factors influencing the outcome in in vitro immunization.

To simplify this approach we established a method excluding immune cells other than the cells needed for antibody production, thereby minimizing the number of factors involved and creating a controllable environment for the in vitro immune response. In the present study, we describe the establishment of this simplified approach in which only B lymphocytes were specifically activated in vitro. In the organism $B$ lymphocytes may be activated by the antigen only ( $T$ cell independent) or by a combination of signals from specifically activated $T$ cells and antigen ( $T$ cell dependent). $T$ cell independent activation of $B$ lymphocytes mostly results in the production of low affinity immunoglobulin (Ig) M antibodies. To evoke the production of specific IgG antibodies, $T$ cells are needed to provide activation signals to the antigen experienced $B$ cell, which comprise the secretion of different cytokines and the expression of the CD40-ligand on the T cell surface (Crotty 2015; Gardell and Parker 2017). In accordance with this in vivo mechanism, we attempt to mimic these activation signals in an in vitro setting to efficiently activate $B$ lymphocytes and induce a specific antibody response. Therefore $B$ cells were isolated from naïve mice and cultured with supplements mimicking the in vivo conditions of antigen-specific activation. B lymphocyte activation was shown in proliferation assays and by 
bioRxiv preprint doi: https://doi.org/10.1101/2020.11.24.393728; this version posted November $24,2020$. The copyright holder for this preprint (which was not certified by peer review) is the author/funder, who has granted bioRxiv a license to display the preprint in perpetuity. It is made available under aCC-BY-NC-ND 4.0 International license.

monitoring surface marker expression. Specific IgM responses in in vitro cultures were detected from day 3 on, specific IgGs from day 9 onwards. By fusion of antigen-specific B lymphocytes with myeloma cells we were able to generate stable hybridoma cell lines secreting antigen-specific IgM and IgG antibodies. To test if the activation protocol is applicable with different types of antigens we challenged murine B cells with heat-inactivated Legionella pneumophila and successfully induced a specific IgG response in our in vitro approach.

\section{Results}

\subsection{Characterization and purity of isolated B lymphocytes}
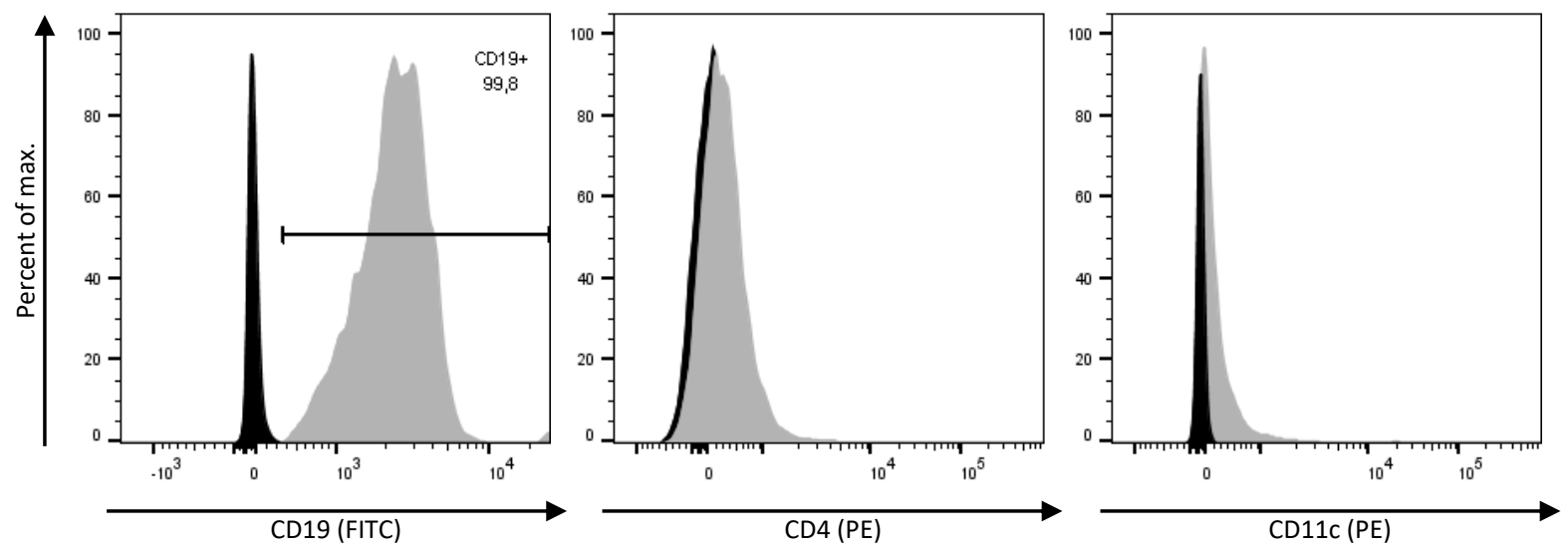

Unstained control

Isolated B lymphocytes
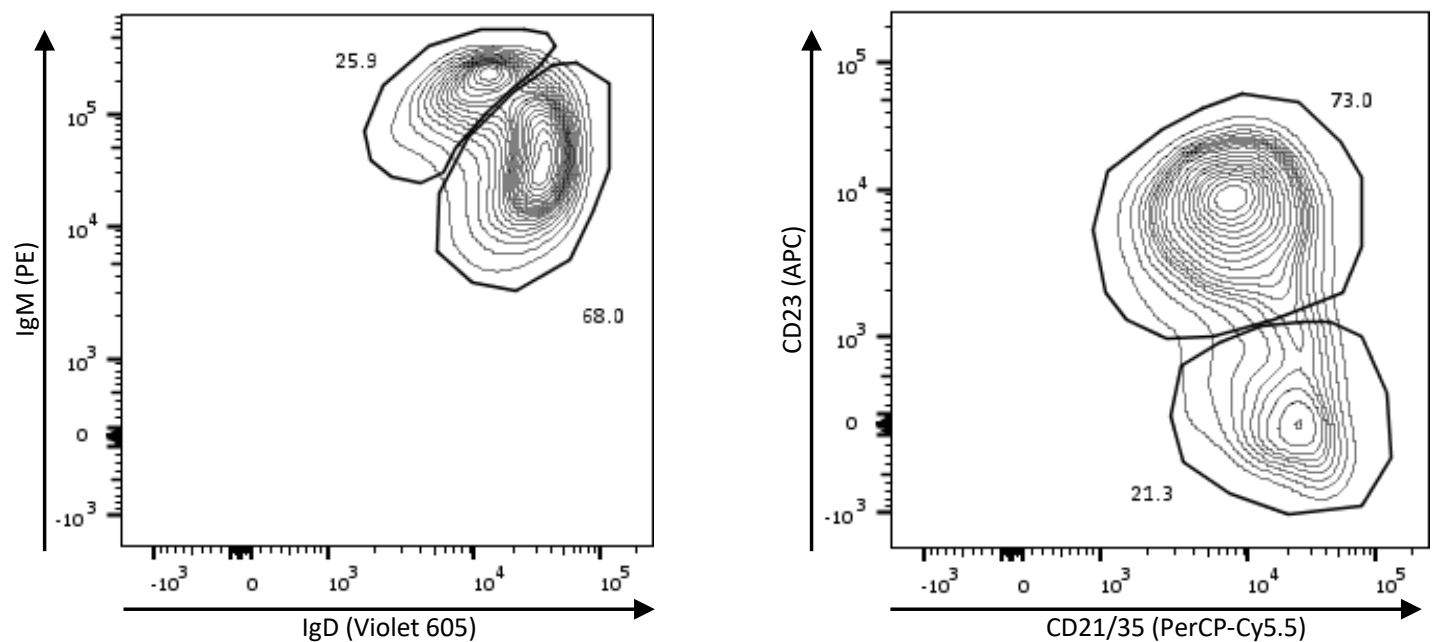

Fig.1: B lymphocytes were isolated from spleen cells of naïve mice by magnetic cell separation. Cells were stained with fluorescein isothiocyanate-labelled anti-CD19, phycoerythrin-labelled anti-CD4, -CD11c and -IgM, Violet 605-labelled anti-IgD, allophycocyanin-labelled anti-CD23 and peridinin chlorophyll-cyanine 5.5-labelled anti-CD21/35 antibodies. By flow cytometric analysis the isolated cells were found

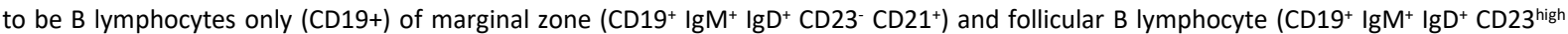
$\mathrm{CD} 21^{+}$) phenotype.

First spleen cells were isolated from naive mice. To eliminate other immune or spleen cells such as T lymphocytes, B lymphocytes were purified through a negative selection with antibody-coated magnetic beads. Purity of B lymphocytes was verified by flow cytometric analysis upon staining CD19, CD4 and CD11C surface markers. All cells were found positive for CD19, but negative for CD4 and CD11c, confirming a pure B lymphocyte population (Fig. 1).

Furthermore we investigated the phenotype of the isolated splenic $B$ cells by analyzing the surface expression of IgD, IgM, CD21/CD35, CD23 in combination with CD19. Flow cytometric analysis revealed 
that all cells expressed IgM, IgD and CD21/35 on their surface, but there were two populations to distinguish. $\operatorname{lgM}^{\text {high }} \operatorname{IgD}{ }^{+}$cells were further found to be $\mathrm{CD}_{23}$, whereas $\lg \mathrm{D}^{\text {high }} \operatorname{IgM}^{+}$cells were shown to be $\mathrm{CD}_{23} 3^{+}$, indicating the phenotypes of marginal zone $\left(\mathrm{CD} 19^{+} \operatorname{IgM}^{+} \operatorname{IgD}{ }^{+} \mathrm{CD} 23^{-} \mathrm{CD} 21^{+}\right)$and follicular $\mathrm{B}$ lymphocytes (CD19 $\left.\operatorname{lgM}^{+} \operatorname{IgD} \mathrm{CD}^{+} 3^{\text {high }} \mathrm{CD} 21^{+}\right)$.

\subsection{Proliferation of B lymphocytes during in vitro stimulation}

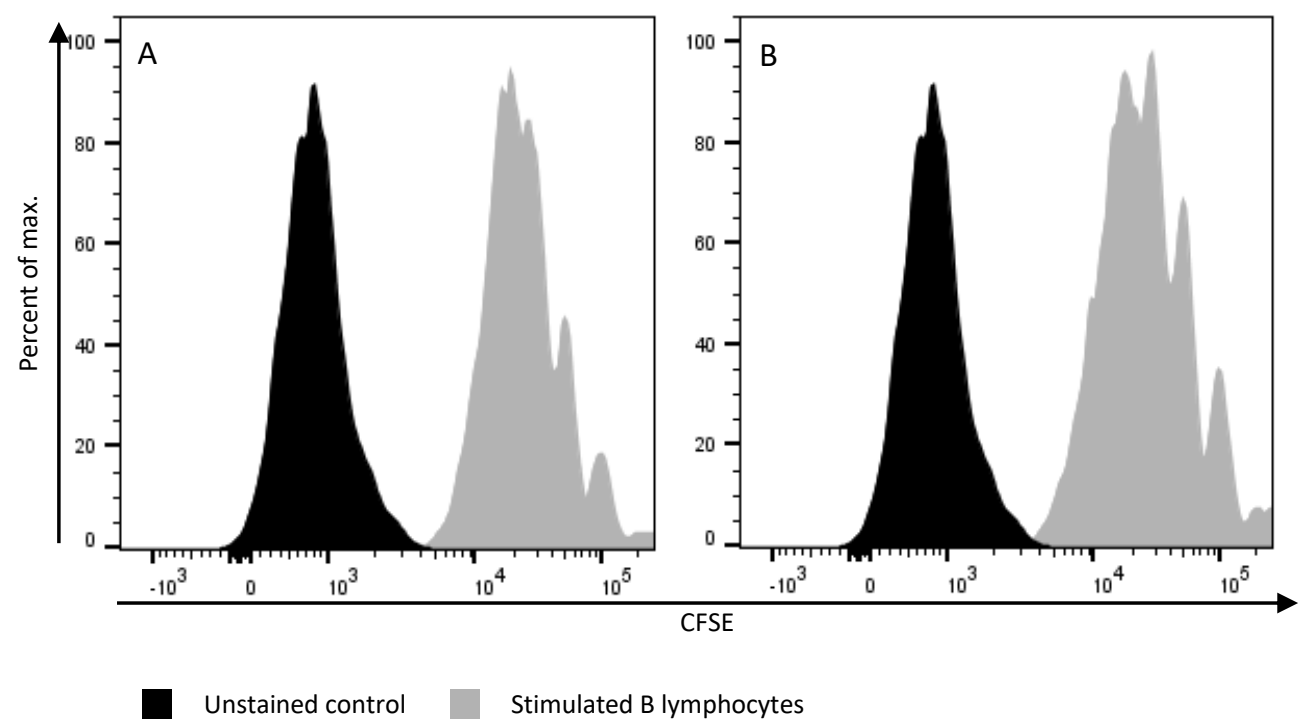

Fig.2: Isolated B lymphocytes were stained with CFSE and stimulated in vitro with $10 \mu \mathrm{g} / \mathrm{ml} \mathrm{VP1} \mathrm{and} 1 \mu \mathrm{g} / \mathrm{ml} \mathrm{LPS}$ (A) or $2 \mathrm{ng} / \mathrm{ml}$ recombinant murine IL7 (B) for ten days. Proliferation as indicated by the decreasing fluorescent intensity of CFSE is shown here on day 7 of in vitro stimulation. In both samples the majority of cells has divided at least three times and a total of four to five daughter generations can be traced.

To induce in vitro B lymphocyte activation, cells were stimulated with a combination of VP1, anti CD40antibody, LPS and IL7 for five days. A subsequent restimulation with the cocktail of stimulants for another five days followed and IL4 was added. To investigate whether B lymphocytes proliferate upon stimulation, isolated B lymphocytes were labelled with CFSE and flow cytometric analysis was performed at different time points. More than $90 \%$ of the cells stimulated with antigen, anti CD40antibody, IL4 and LPS or IL7 proliferated upon stimulation. By day 7 the majority of stimulated B lymphocytes had divided at least three times as shown in Fig. 2. Proliferation however seemed to decline after day 7 as there is no considerable difference seen between proliferation status of cultures from day 7 and day 9 (not shown). Number of living cells did not increase beyond input numbers but stayed roughly constant throughout the stimulation. Unstimulated control cells did not show any proliferation. Also stimulation with antigen only had no effect on B cell growth. In fact these cultures constantly decreased in numbers of living cells and cells had eventually died out by day 9 . 


\subsection{Activation of B lymphocytes}

Upon activation of $B$ lymphocytes the surface marker expression changes. We therefore examined the stimulated cells for their expression patterns throughout the stimulation. Surface markers CD69, CD80, CD86, CD138 and surface IgG were labelled and analyzed at different time points. Stimulated cells showed a drift towards higher expression of activation markers CD69, CD80 and CD86 within ten days indicating a successful in vitro activation (Fig. 3). A fraction of the isolated cells already expressed surface IgG at an intermediate level initially. After ten days of in vitro stimulation the expression had shifted to a considerably higher level in all cells. However, the expression of CD138 as a prominent marker of plasma cells did not or only marginally increase.
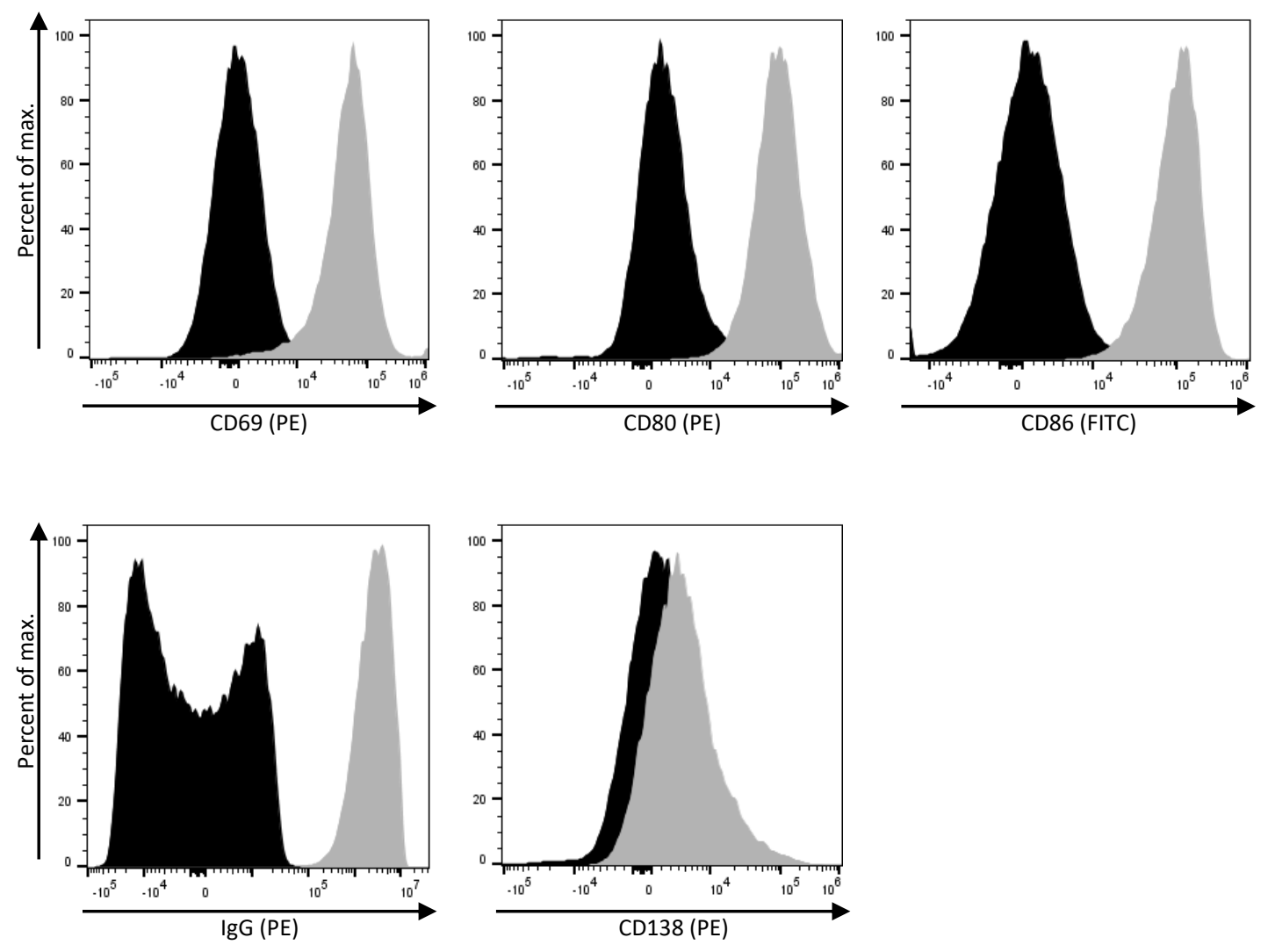

Unstimulated B lymphocytes (day 0)

Stimulated B lymphocytes (day 10)

Fig.3: In vitro stimulated B lymphocytes were stained for expression of surface activation markers CD69, CD80, CD86, CD138 and surface IgG. Compared to unstimulated cells a strong increase in $\mathrm{CD} 69, \mathrm{CD} 80, \mathrm{CD} 86$ and surface IgG expression was observed in cultures stimulated with antigen (VP1), anti CD40-antibody, IL4, LPS or IL7. Expression of CD138 did not change remarkably within the ten days of in vitro stimulation.

\subsection{Induction of antigen-specific antibodies by in vitro activation}

During stimulation, the presence of antibodies specific for the antigen VP1 among the different culture settings was determined daily by ELISA (Fig. 4). Specific IgM antibodies were detected from day 3 on in cultures stimulated with antigen alone or antigen, anti CD40-antibody, IL4, LPS or IL7. Specific IgG secretion only evoked upon secondary antigen stimulation and peaked on day 10 of in vitro activation similar to IgM. In stimulated cultures we detected a significantly higher level of VP1-specific IgM and IgG antibodies compared to unstimulated control cultures. While cells stimulated with antigen alone showed highest levels of both specific IgM and IgG these cells suffered from poor viability and did not proliferate. These data show a successful activation of naive B lymphocytes in vitro induced by signals mimicking in vivo $\mathrm{T}$ cell activation. 

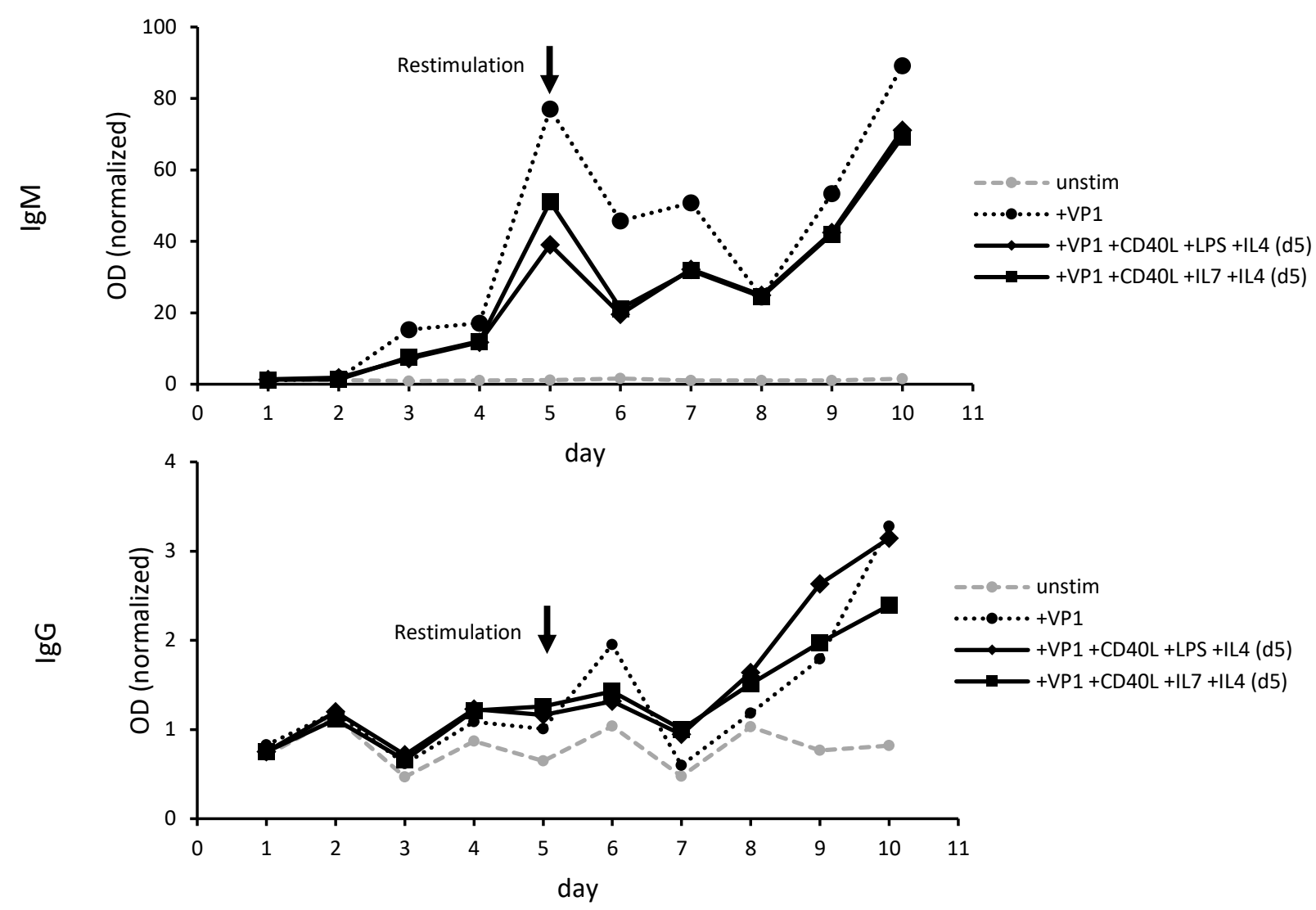

Fig.4: Murine B lymphocytes isolated from naive mice were stimulated in vitro with antigen (VP1), anti CD40-antibody, IL4, LPS or IL7 and restimulated after five days. Supernatants were tested daily for specific IgM and IgG antibodies. A strong IgM response arose from day 3 onwards in samples stimulated with antigen (VP1), anti CD40-antibody, IL4, LPS or IL7 and peaked in a signal 90-fold higher than that of unstimulated cells on day 10. Antigen-specific IgG antibodies were detected on day 9 in the same samples.

\subsection{Establishment of a monoclonal antibody with in vitro activation}

We then attempted to establish a monoclonal antibody by using our activation method in combination with hybridoma technology. Fusion to generate hybridomas from the in vitro activated B lymphocytes was carried out five days after restimulation, i.e. after ten days of in vitro activation. Fusion efficiency was very high among cultures stimulated with antigen, anti-CD40 antibody, IL4 and LPS or IL7 and several clones could be established. After the selection process we identified several monoclonal hybridoma cell lines stably producing VP1-specific IgG antibodies of IgG2b subclass (Fig. 6). For all antibodies derived from in vitro immunization positive signals $(O D>0.2)$ were obtained at concentrations of $0.625 \mu \mathrm{g} / \mathrm{ml}$ or higher, however signal intensity is lower compared to an antibody (P157) obtained from conventional in vivo immunization with VP1 (Fig. 5). 


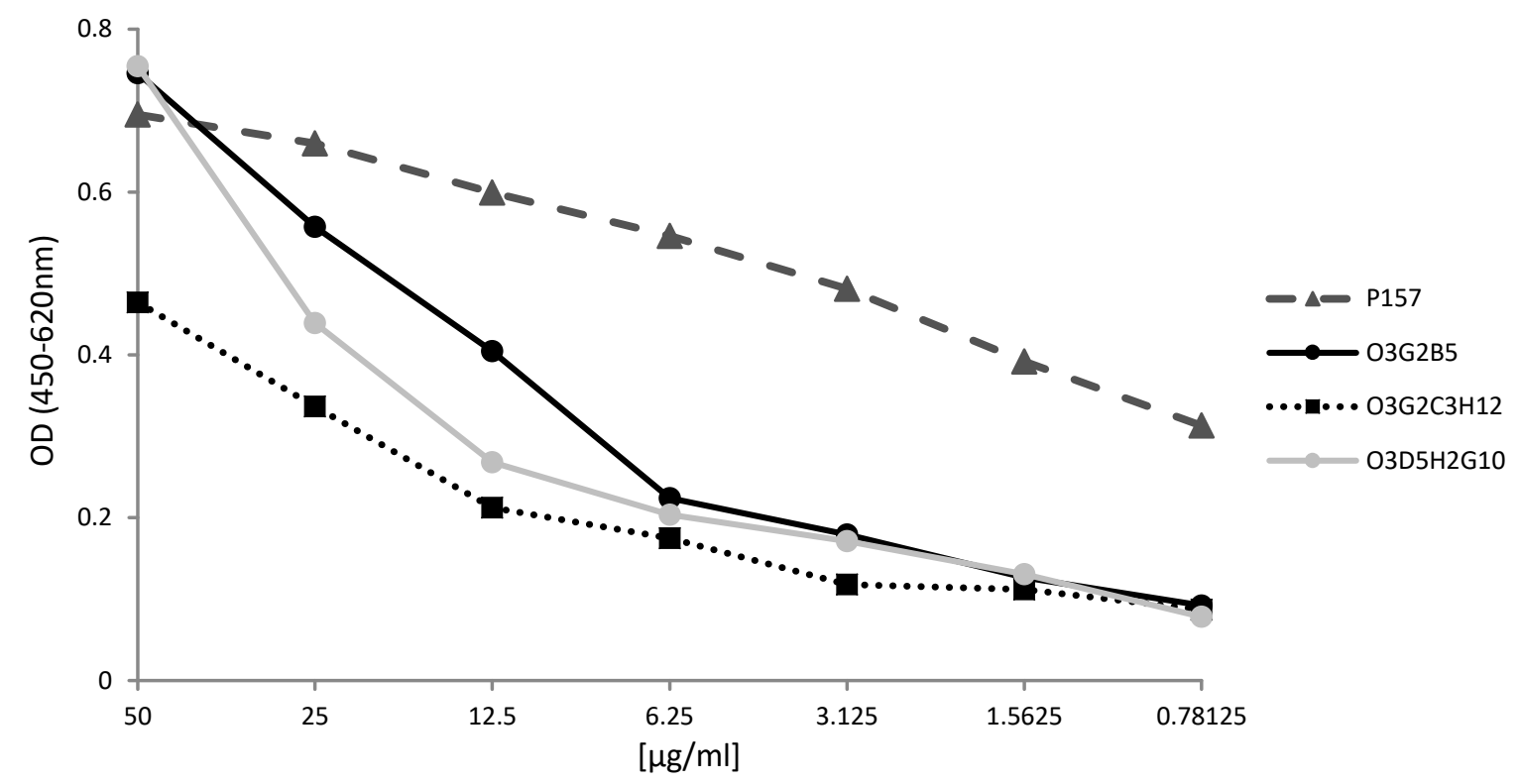

Fig.5: Purified monoclonal antibodies were tested against the antigen VP1. Antibodies O3G2B5, O3D5H2G10 and 03G2C3H12 were obtained from generated hybridoma cell lines after in vitro immunization of B lymphocytes with O3G2B5 showing the highest binding ability against the antigen. In comparison, P157 is an antibody derived from conventional in vivo immunization with VP1. Antibody concentrations ranged from 50 to $0.78125 \mu \mathrm{g} / \mathrm{ml}$.

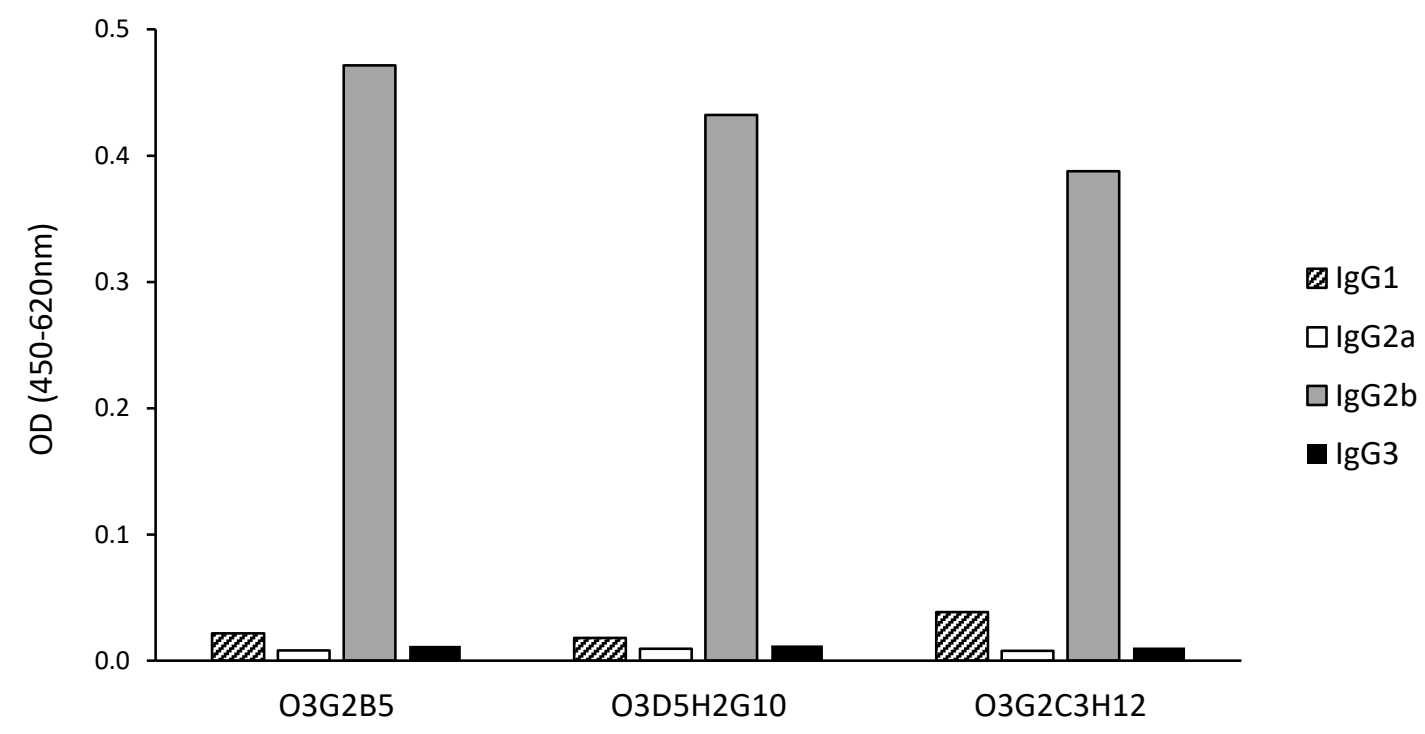

Fig.6: Subclasses of purified monoclonal antibodies were determined by ELISA. Subclasses were detected by subclass specific biotin-labeled antibodies and subsequent streptavidin-conjugated horse radish peroxidase. All antibodies were identified as IgG2b.

\subsection{Induction of in vitro antibody response against Legionella pneumophila}

Next we challenged murine B lymphocyte cultures with heat-inactivated L. pneumophila to test transferability of the activation protocol to different antigen types. Culture supernatants were tested for the presence of specific IgG antibodies by flow cytometric analysis. Therefore L. pneumophila were incubated with the supernatants of stimulated versus unstimulated B lymphocytes and subsequently stained with a PE-labelled anti-mouse IgG secondary antibody. Supernatants from cultures stimulated 
with both L. pneumophila, anti CD40-antibody, IL4 and LPS or IL7 (Fig. 7) clearly contained IgG antibodies capable of binding to and labelling L. pneumophila whereas unstimulated B lymphocyte cultures did not produce such.
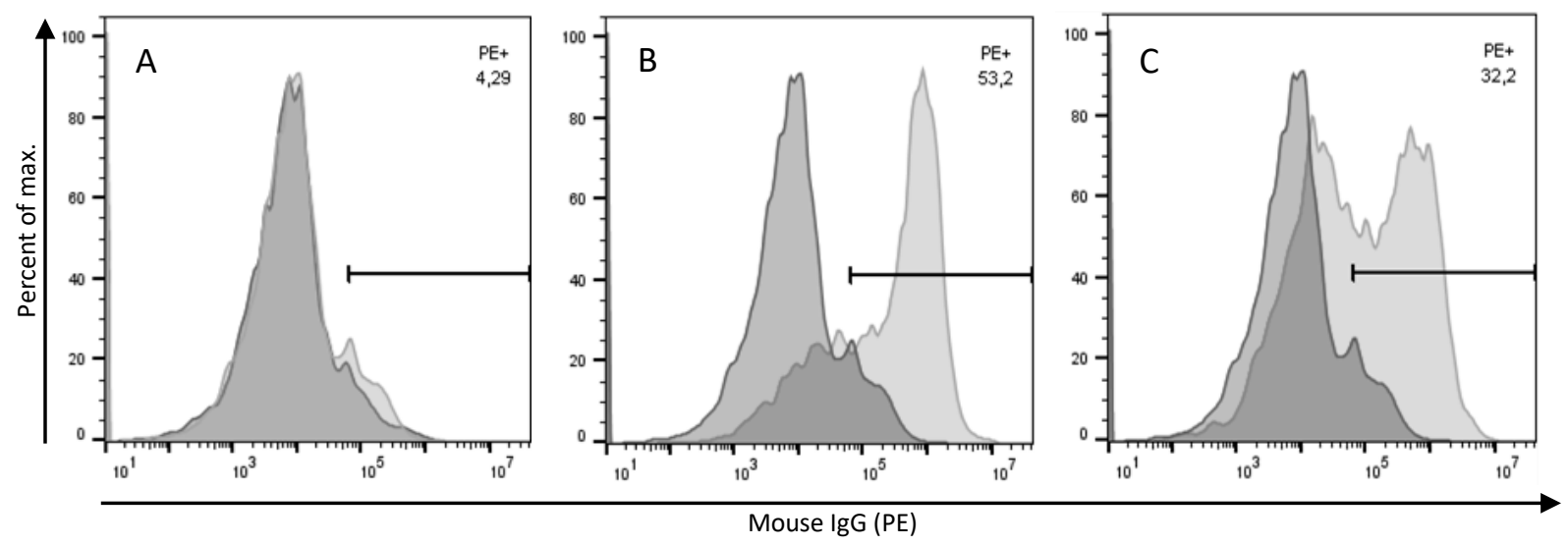

L. pneumophila incubated with PE-labelled anti-mouse IgG

L. pneumophila incubated with B lymphocyte culture supernatant and PE-labelled anti-mouse IgG subsequently

Fig.7: Specific IgG antibodies against L. pneumophila by in vitro activation of B lymphocytes. Murine B lymphocytes from naive mice were cultured in vitro with or without heat-inactivated $L$. pneumophila and a combination of stimuli and restimulated after five days. After ten days of in vitro activation supernatants from B lymphocyte cultures were harvested and incubated with heat-inactivated $L$. pneumophila. Upon washing L. pneumophila were stained with PE-labelled anti-mouse IgG secondary antibody. A: L. pneumophila incubated with supernatant from unstimulated murine naïve B lymphocytes. B and C: L. pneumophila incubated with supernatants from B lymphocytes that were stimulated with heat-inactivated L. pneumophila, anti CD40-antibody, IL4 and LPS (B) or IL7 (C), respectively. Samples labelled with secondary anti-mouse IgG antibody only are depicted in dark grey; shown in light grey are samples incubated with supernatant and subsequently with secondary antibody.

\section{Discussion}

In this study we showed the in vitro induction of a specific IgG antibody response by in vitro activation of murine B lymphocytes. For the activation of B lymphocytes in vivo a coordinated action of DCs and $T$ lymphocytes is necessary to trigger a specific IgG response by B lymphocytes. In vivo, DCs present antigen to $T$ and probably also $B$ lymphocytes. Since B lymphocytes also function as antigen presenting cells, they might take over antigen presentation themselves in vitro instead of DCs (Rodríguez-Pinto, 2005). Whether specific subsets of $B$ lymphocytes are responsible for this function is yet unknown and has to be clarified (Chen and Jensen, 2008; den Haan et al., 2014; Hong et al., 2018; Kambayashi and Laufer, 2014). In vivo, T lymphocytes take up processed antigen from DCs and present a complex of MHCs and antigenic peptides to B lymphocytes. In combination with a range of further signals, this process activates antigen-specific $B$ lymphocytes to produce antibodies whose subclass depends on these signals. T lymphocytes are also partially responsible for the avoidance of an immune response against the body's own antigens. As far as we can consider, autoimmune reactions do not play a role during in vitro in contrast to in vivo immunization, thus allow for a wider spectrum of antibodies.

The main obstacle in in vitro immunization is finding a method to activate $B$ lymphocytes to produce antibodies of the wanted subclass and specificity. In vivo, B lymphocytes can be activated in a T cellindependent manner by antigen only leading to the production of low affinity IgM antibodies. This was transferred to in vitro settings repeatedly (Ait Mebarek et al., 2013; Federspiel et al., 1991; Wohlleben et al., 1996). Those methods however lack an efficient activation of $B$ cells resulting in a limited number of sufficiently matured antibody producing cells, and thus struggle to deliver specific antibodies other than IgM. Other studies succeeded to induce antigen specific IgG responses in vitro by immunizing a heterogeneous population of splenocytes by exposure to antigen only or with a combination of cytokines and stimulative antibodies (Ravi et al., 2007; Inagaki et al., 2013), but failed to deliver a 
reliable and reproducible protocol presumably due to variation in antigen specific and successfully activated immune cell populations. To overcome these difficulties we aimed to reduce the impact of variables. In the present study we therefore tried to specifically activate murine B lymphocytes alone in vitro.

Splenic B lymphocytes were isolated from naive $\mathrm{C} 57 \mathrm{BI} / 6$ mice and purity was shown by FACS analysis as no DCs or T cells could be detected in the cell suspension. In vivo signals of antigen-specific $\mathrm{T}$ cell mediated activation were mimicked in vitro by using a range of immunomodulators in different combinations. To circumvent the DC function, B lymphocytes were challenged with a surplus of soluble model antigen VP1. B cell activation in vitro was verified by changes in surface receptor expression, proliferation and antibody production. Indeed, the addition of anti CD40-antibody, IL4 and LPS or IL7 to the culture medium in combination with the antigen led to an efficient activation of B lymphocytes in vitro and moreover resulted in an increased production of anti-VP1 IgG antibodies by the B lymphocytes. Furthermore we were able to equivalently induce specific IgG responses when activating $B$ lymphocytes in vitro in similar settings with other antigens such as $L$. pneumophila. Without $T$ cellsubstituting ingredients the specific IgG response was significantly lower as was cell viability. Moreover, it was not possible to sustain living cells throughout the stimulation to generate hybridoma cells from. From B lymphocyte cultures supplemented with antigen and T cell-replacing ingredients we were able to produce a number of stable hybridomas synthesizing VP1 specific antibodies of IgG2b subclass. Hence, it is proven possible to obtain monoclonal antigen-specific antibodies with our in vitro method. While antibodies generated in in vitro immunization produced positive ELISA signals at low concentrations, overall signal intensities were lower compared to an anti VP1 antibody obtained from conventional in vivo immunization. This can be attributed to the shortened time period of immunization and thus incomplete affinity maturation. In contrast to the conventional method of antibody production by in vivo immunization the procedure was shortened from several months to a few days while lower amounts of antigen were needed and potential distress for experimental animals was minimized.

Several aspects require further exploration. Since VP1 as a viral protein has adjuvant effects it has to be clarified whether other proteins and haptens can induce an in vitro antibody response in the same setting. Additionally, the method is to be tested for the human system which could be a substantial breakthrough. Eventually, this may be a universal procedure to produce specific antibodies in vitro minimally invasive from various species.

\section{Material and methods}

\subsection{Purification and culture of murine splenic B lymphocytes}

Spleens were removed from naïve C57BI/6j mice (8-12 weeks). Single cell suspensions of splenocytes were obtained by dispersion through a $40 \mu \mathrm{M}$ cell strainer (VWR, Radnor, Pennsylvania, USA). B cells were isolated by negative selection using the B Cell Isolation Kit (Miltenyi Biotec, Bergisch-Gladbach, Germany). The procedure was carried out following the instructions of the manufacturer. Purified B cells were cultured in 24 well plates (TPP, Trasadingen, Switzerland) at $10^{6}$ cells/well in $1 \mathrm{ml} \mathrm{RPMI} 1640$ complete medium (Thermo Fisher Scientific, Waltham, Massachusetts, USA) supplemented with $10 \%$ heat-inactivated fetal calf serum (FCS, Thermo Fisher Scientific, Waltham, Massachusetts, USA), 2 mM L-glutamine (Roth, Karlsruhe, Germany) and $50 \mu \mathrm{M}$ 2-mercaptoethanol (Roth, Karlsruhe, Germany) and kept at $37{ }^{\circ} \mathrm{C}$ and $5 \% \mathrm{CO}_{2}$. 


\subsection{Proliferation assay}

Following purification, B lymphocytes were stained with carboxyfluorescein succinimidyl ester (CFSE, Thermo Fisher Scientific, Waltham, Massachusetts, USA) according to the protocol published by Quah et al. (2007). In short, cells were resuspended in PBS containing $5 \%$ FCS. CFSE was added to a final concentration of $1.25 \mu \mathrm{M}$ and cell suspension was mixed immediately. Cells were left to incubate for $5 \mathrm{~min}$ at room temperature (RT) in the dark. Subsequently, cells were washed three times by diluting the suspension with PBS/5\% FCS and centrifugation was performed for 5 min at $300 \times$ g. Cells were resuspended in complete medium and used for in vitro stimulation. Before analysis, cells were stained with $1 \mu \mathrm{M}$ PoPro-1 lodide (Thermo Fisher Scientific, Waltham, Massachusetts, USA) for $10 \mathrm{~min}$ at $4{ }^{\circ} \mathrm{C}$ in the dark to discriminate living cells. Cells were examined using the Attune Flow Cytometer (Thermo Fisher Scientific, Waltham, Massachusetts, USA). Data were analysed using the FlowJo software (FlowJo LLC, Ashland, Oregon, USA).

\subsection{In vitro immunization}

Following purification or CFSE staining, naïve B lymphocytes were challenged with $10 \mu \mathrm{g} / \mathrm{ml}$ hamster polyomavirus capsid protein (VP1, as described previously in Lütkecosmann et al., 2019) serving as model antigen or $5 \times 10^{4}$ to $5 \times 10^{6}$ heat-inactivated Legionella pneumophila (kindly gifted by sifin diagnostics gmbh, Berlin, Germany) per ml. Different combinations of stimuli were added to the culture, i.e. $2 \mu \mathrm{g} / \mathrm{ml}$ rat anti-mouse CD40 antibody (BioLegend, San Diego, California, USA), $2 \mathrm{ng} / \mathrm{ml}$ interleukin 7 (IL7) (Miltenyi Biotec, Bergisch-Gladbach, Germany) and/or $1 \mu \mathrm{g} / \mathrm{ml}$ lipopolysaccharide (LPS) (Merck KGaA, Darmstadt, Germany). Control cultures were left untreated. Culture supernatants were collected for the determination of specific antibodies. On day 5 of cultivation, remaining supernatant was reduced to $500 \mu \mathrm{l}$. To restimulate the cells, $1 \mathrm{ml}$ of medium containing VP1 or inactivated L. pneumophila and the appropriate stimuli was added to each well. Additionally, interleukin 4 (IL4) (Miltenyi Biotec, Bergisch-Gladbach, Germany) was added to some wells at $10 \mathrm{ng} / \mathrm{ml}$.

\subsection{Flow cytometric analysis of B lymphocytes}

Activation status of B lymphocytes was examined by flow cytometric analysis of several surface activation markers. Therefore, cells were harvested and washed in PBS. Cells were then labelled in $300 \mu \mathrm{l}$ staining buffer using combinations of different antibodies for $30 \mathrm{~min}$ at $4{ }^{\circ} \mathrm{C}$ in the dark (antimouse CD4, CD69 (BD Bioscience, Franklin Lakes, New Jersey, USA), CD19, IgD (Thermo Fisher Scientific, Waltham, Massachusetts, USA), CD11c, CD80, CD86, CD138 (Miltenyi Biotec, BergischGladbach, Germany), CD21/CD35, CD23, IgM and IgG (BioLegend, San Diego, California, USA)). Cells were then washed as before and resuspended in $300 \mu \mathrm{l} 1 \mu \mathrm{M}$ PoPro-1 lodide to obtain the total number of living cells. Upon incubation for $10 \mathrm{~min}$ at $4{ }^{\circ} \mathrm{C}$ in the dark, cells were examined using the BD FACSAria III (BD Bioscience, Franklin Lakes, New Jersey, USA). Data were analysed using the FlowJo software.

\subsection{Detection of antigen-specific antibodies}

Supernatants were tested daily in enzyme-linked immunosorbent assay (ELISA) for specific IgM and IgG production. Therefore, 96 well plates were coated with $50 \mu \mathrm{l} /$ well of $5 \mu \mathrm{g} / \mathrm{ml}$ antigen in PBS at $4{ }^{\circ} \mathrm{C}$ overnight. Following blocking with $100 \mu \mathrm{l}$ PBS containing $5 \%$ neonatal calf serum (NCS) for $60 \mathrm{~min}$ at $\mathrm{RT}, 50 \mu \mathrm{l}$ culture supernatants were added for $45 \mathrm{~min}$ at RT. For the detection of antigen-bound antibodies the wells were incubated with $50 \mu$ l of horseradish peroxidase (HRP)-labeled anti-mouse IgM or IgG antibodies (Dianova, Hamburg, Germany) for $45 \mathrm{~min}$ at RT. Finally, $50 \mu$ l substrate solution 
containing $0.12 \mathrm{mg} / \mathrm{ml}$ tetramethylbenzidine (Roth, Karlsruhe, Germany, in $50 \mathrm{mM} \mathrm{NaH} \mathrm{PO}_{4}$ with $0.04 \% \mathrm{CH}_{4} \mathrm{~N}_{2} \mathrm{O} \cdot \mathrm{H}_{2} \mathrm{O}_{2}$ ) were added per well and the reaction was stopped using $1 \mathrm{M}$ sulfuric acid. Absorbance was measured at $450 \mathrm{~nm}$ with a reference wavelength of $620 \mathrm{~nm}$ using the Multiskan FC Microplate Photometer (Thermo Fisher Scientific, Waltham, Massachusetts, USA).

\subsection{Cell fusion of murine B lymphocytes and purification of monoclonal antibodies}

On day 10 of in vitro culture and stimulation, cells were harvested for fusion with murine myeloma cell line SP2/0-Ag14 by electrofusion in the presence of polyethylene glycol (Roth, Karlsruhe, Germany) as described previously (Holzlöhner and Hanack 2017). Fused cells were then seeded on 96 well flat bottom plates onto mouse peritoneal cells as feeder layer in hypoxanthine-aminopterine-thymidine (VWR, Radnor, Pennsylvania, USA) medium for the selection process.

Cultures with positive ELISA signals were seeded in limited dilution and inspected microscopically for monoclonal growth. Monoclonal hybridoma were transferred into T75-flasks (VWR, Radnor, Pennsylvania, USA) and supernatants were collected up to $500 \mathrm{ml}$. IgG antibodies were then purified as described previously (Holzlöhner and Hanack 2017). In short, supernatants were filtered through a $0.45 \mu \mathrm{m}$ filter (Sartorius Stedim Biotech $\mathrm{GmbH}$, Goettingen, Germany), mixed with binding buffer (4 M $\mathrm{NaCl}$ and $1 \mathrm{M}$ glycine $\mathrm{NaOH}, \mathrm{pH} 8.9$ ) and then loaded onto a protein A column. Bound antibodies were eluted with $0.1 \mathrm{M}$ citrate, $\mathrm{pH} 3.5$, and neutralized with $500 \mu \mathrm{l} \mathrm{Tris-} \mathrm{HCl}, \mathrm{pH} 9.0$.

\subsection{Determination of immunoglobulin subclass and direct ELISA}

ELISA was carried out as described before. Briefly, 96 well plates were coated with $50 \mu \mathrm{l} /$ well of $5 \mu \mathrm{g} / \mathrm{ml}$ antigen in PBS at $4{ }^{\circ} \mathrm{C}$ overnight. Following blocking with $100 \mu \mathrm{l} \mathrm{PBS}$ containing $5 \%$ NCS, $50 \mu \mathrm{l}$ of antibody diluted in PBS $/ 5 \%$ NCS $(50-0.78 \mu \mathrm{g} / \mathrm{ml})$ were added for $45 \mathrm{~min}$ at RT. To determine subclasses of purified antibodies wells were incubated for $45 \mathrm{~min}$ with $50 \mu \mathrm{l}$ of biotin-labeled antimouse IgG1, IgG2a, IgG2b or IgG3 antibodies (Jackson ImmunoResearch Europe Ltd, Cambridgeshire, United Kingdom). Subsequently, $50 \mu$ l of streptavidin-HRP (Merck KGaA, Darmstadt, Germany) were added and incubated for $45 \mathrm{~min}$. For comparison of antibodies, wells were incubated with $50 \mu \mathrm{l}$ of HRP-labeled anti-mouse IgG antibodies (Dianova, Hamburg, Germany) for $45 \mathrm{~min}$ at RT. Subsequent steps were carried out as described before.

\subsection{Detection of antibodies specific for Legionella pneumophila}

Heat inactivated L. pneumophila were washed with PBS at $6500 \times \mathrm{g}$ for 5 min and incubated with $100 \mu \mathrm{l}$ supernatant from stimulated or unstimulated B lymphocyte cultures. Upon washing, L. pneumophila were incubated with PE-labelled anti-mouse IgG antibodies for $30 \mathrm{~min}$ at $4{ }^{\circ} \mathrm{C}$ in the dark. L. pneumophila were then washed as before and examined using the Attune Flow Cytometer (Applied Biosystems, Foster City, California, USA). Flow cytometric data were analysed using the FlowJo software.

\section{Acknowledgements}

We thank the German Federal Ministry of Education and Research for funding the work (03IPT7030X). 
Competing interests

The corresponding author Katja Hanack is owner and manager of new/era/mabs. The other authors declare no competing financial or non-financial interests.

\section{References}

Ait Mebarek, M., Wijkhuisen, A., Adel-Patient, K., Lamourette, P., Léonetti, M., Volland, H., 2013. Production of human antibodies by in vitro immunization using a fusion protein containing the transcriptional transactivator of HIV-1. Journal of Immunological Methods 396, 96-106. https://doi.org/10.1016/j.jim.2013.07.015

Borrebaeck, C.A., Danielsson, L., Moller, S.A., 1988. Human monoclonal antibodies produced by primary in vitro immunization of peripheral blood lymphocytes. Proceedings of the National Academy of Sciences 85, 3995-3999. https://doi.org/10.1073/pnas.85.11.3995

Chen, X., Jensen, P.E., 2008. The role of B lymphocytes as antigen-presenting cells. Arch. Immunol. Ther. Exp. 56, 77-83. https://doi.org/10.1007/s00005-008-0014-5

Crotty, S., 2015. A brief history of T cell help to B cells. Nat Rev Immunol 15, 185-189. https://doi.org/10.1038/nri3803

den Haan, J.M.M., Arens, R., van Zelm, M.C., 2014. The activation of the adaptive immune system: Cross-talk between antigen-presenting cells, T cells and B cells. Immunology Letters 162, 103-112. https://doi.org/10.1016/j.imlet.2014.10.011

Federspiel, G., McCullough, K.C., Kihm, U., 1991. Production of monoclonal antibodies specific for African swine fever virus following in vitro primary immunization of mouse splenocytes in the presence of stimulated T lymphocyte supernatants. Journal of Immunological Methods 145, 71-81. https://doi.org/10.1016/0022-1759(91)90312-4

Gardell, J.L., Parker, D.C., 2017. CD40L is transferred to antigen-presenting B cells during delivery of T-cell help: CD40L is transferred to antigen-presenting. Eur. J. Immunol. 47, 41-50. https://doi.org/10.1002/eji.201646504

Geskin, L.J., 2015. Monoclonal Antibodies. Dermatologic Clinics 33, 777-786. https://doi.org/10.1016/j.det.2015.05.015

Holzlöhner, P., Hanack, K., 2017. Generation of Murine Monoclonal Antibodies by Hybridoma Technology. JoVE 54832. https://doi.org/10.3791/54832

Hong, S., Zhang, Zhimin, Liu, H., Tian, M., Zhu, X., Zhang, Zhuqiang, Wang, W., Zhou, X., Zhang, F., Ge, Q., Zhu, B., Tang, H., Hua, Z., Hou, B., 2018. B Cells Are the Dominant Antigen-Presenting Cells that Activate Naive CD4+ T Cells upon Immunization with a Virus-Derived Nanoparticle Antigen. Immunity 49, 695-708.e4. https://doi.org/10.1016/j.immuni.2018.08.012

Inagaki, T., Yoshimi, T., Kobayashi, S., Kawahara, M., Nagamune, T., 2013. Analysis of cellular phenotype during in vitro immunization of murine splenocytes for generating antigenspecific immunoglobulin. Journal of Bioscience and Bioengineering 115, 339-345. https://doi.org/10.1016/j.jbiosc.2012.10.008

Kambayashi, T., Laufer, T.M., 2014. Atypical MHC class II-expressing antigen-presenting cells: can anything replace a dendritic cell? Nat Rev Immunol 14, 719-730. https://doi.org/10.1038/nri3754 
Kato, M., Yan, H., Tsuji, N.M., Chiba, T., Hanyu, Y., 2012. A method for inducing antigen-specific IgG production by in vitro immunization. Journal of Immunological Methods 386, 60-69. https://doi.org/10.1016/j.jim.2012.08.019

Lütkecosmann, S., Faupel, T., Porstmann, S., Porstmann, T., Micheel, B., Hanack, K., 2019. A crossreactive monoclonal antibody as universal detection antibody in autoantibody diagnostic assays. Clinica Chimica Acta 499, 87-92. https://doi.org/10.1016/j.cca.2019.09.003

Mishell, R.I., Dutton, R.W., 1966. Immunization of Normal Mouse Spleen Cell Suspensions in vitro. Science 153, 1004-1006. https://doi.org/10.1126/science.153.3739.1004

Quah, B.J.C., Warren, H.S., Parish, C.R., 2007. Monitoring lymphocyte proliferation in vitro and in vivo with the intracellular fluorescent dye carboxyfluorescein diacetate succinimidyl ester. Nat Protoc 2, 2049-2056. https://doi.org/10.1038/nprot.2007.296

Ravi, M., Sundar, S.S., Kumar, K.K., Parvathi, D., Paul, S.F.D., 2007. Hybridoma Generation by In Vitro Immunization of Murine Splenocytes with Cytosolic Proteins of Chinese Hamster Ovary (CHO) Mitotic Cells. Hybridoma 26, 311-315. https://doi.org/10.1089/hyb.2007.0506

Rodríguez-Pinto, D., 2005. B cells as antigen presenting cells. Cell. Immunol. 238, 67-75. https://doi.org/10.1016/j.cellimm.2006.02.005

Wand, I., Holzlöhner, P., Neupert, S., Micheel, B., Heilmann, K., 2011. Cooperation of dendritic cells with naïve lymphocyte populations to induce the generation of antigen-specific antibodies in vitro. Journal of Biotechnology 156, 173-181. https://doi.org/10.1016/j.jbiotec.2011.09.002

Wohlleben, G., Gray, D., Schimpl, A., 1996. In vitro immunization of naive mouse B cells: establishment of IgM secreting hybridomas specific for soluble protein or hapten from B cells cultured on CD4O ligand transfected mouse fibroblasts. Int Immunol 8, 343-349. https://doi.org/10.1093/intimm/8.3.343 